\title{
Sizing Inductors of Linear Generator for CAD
}

\author{
D. Lahouel*, D. Hedjazi, R. Abdessemed \\ Department of Electrical Engineering, University of Batna, Algeria.
}

\begin{abstract}
This paper presents a design method of inducing a linear generator in a form of a Cycloid Pendulum, this method solves the problem of the distribution of magnetic field lines in the armature coils and the induction generator, observe the shape of the magnetic field created by the coil, measure the dependencies of field with the position along its axis and the current density injected, and that by the Finite Element and is used the Computer-Aided Design (CAD), and more particularly the Comsol Multiphysics software to get the results that good operation of the generator.
\end{abstract}

Keywords: «CAD», «Comsol Multiphysics», «Finite Elements», «Linear Generator», «Magnetic Vector Potential», «Sizing of Coils»

\section{Introduction}

The electrical machine (rotary or linear) during operation under the action of electromagnetic fields. In any machine the coil or coils produces a magnetic field constant or variable over time and often in space.

Knowledge of the field allows access to computing performance and operation of the device in permanent and transient, and evaluation of the field at each point in space yet possible to deduce the flow and so the electromotive forces for operation as a generator or couple to the engine operation.

In many stages of the design process of an electrical device, the evaluation of electric and magnetic fields appear as a necessary work, and from that, we analyze the relationship between the maximum field strength and the geometrical dimensions, and a comparison was made between dependency of the place of the theoretical and the measured location [1], [2].

So in this paper, we study the coil (size, current density, magnetic field, electromotive force) since it is the main component of the inductor of the linear generator that we study later.

An important aspect of survival, it is the complexity and longevity of the technology.

More complex a system is and the more moving parts which compose it, the more it is likely that some will fail. In addition, to improve the chances of becoming commercially attractive, the need for maintenance must be kept to a minimum. To meet these requirements, the computer-aided design a very important step to good order and avoid all the difficulties that may be encountered in the actual design.

The system comprises two reels, whose excitation of the one cause the displacement of the core assembled with a ferromagnetic part in a direction. the median position is ensured by an external cause which is an external power in our work we have modeled mathematically operation of linear generator which consists of a series of coils moving inside the stator, this circuit of coils is then fed from a DC voltage source.

Actuators for longitudinal displacement, we frequently encounter three topologies:

- The armature is movable and the inductor is fixed as shown in figure (1. a). The inductor is secured to the rail so fixed also. The armature is moving and the support board or power supply that is connected thereto by a flexible cable which limits the stroke of its movement.

- The armature is fixed and the inductor, the fixed rail is movable as indicated in the figure (1. b).

- Inductor mobile and stationary armature (as it is distributed over the length of a rail) as the following diagram (Fig. 1. c) indicates.

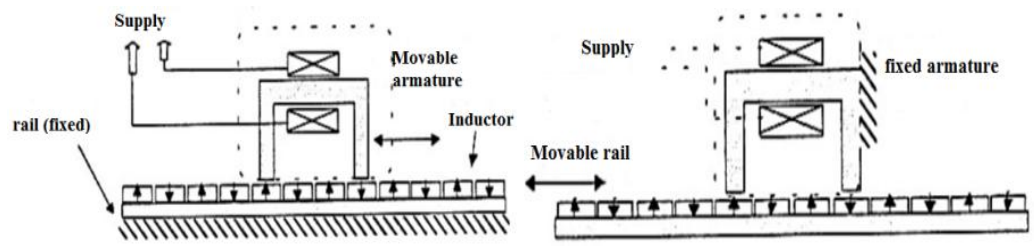

(a)

(b)

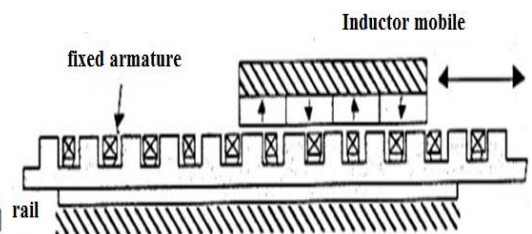

(c)

Fig. 1: Topologies of actuators for longitudinal displacement

We remain on a purely technical level as interest in this type of training is explained through its technology:

- High dynamic motion (speed, acceleration)

- High precision positioning 
- High reliability, longer service life

- Flexibility of use (independent party on the same axis) [3].

\section{Shape Linear Generator} indicated:

In this example, a linear generator in a form of a Cycloid Pendulum as in the figure below we study
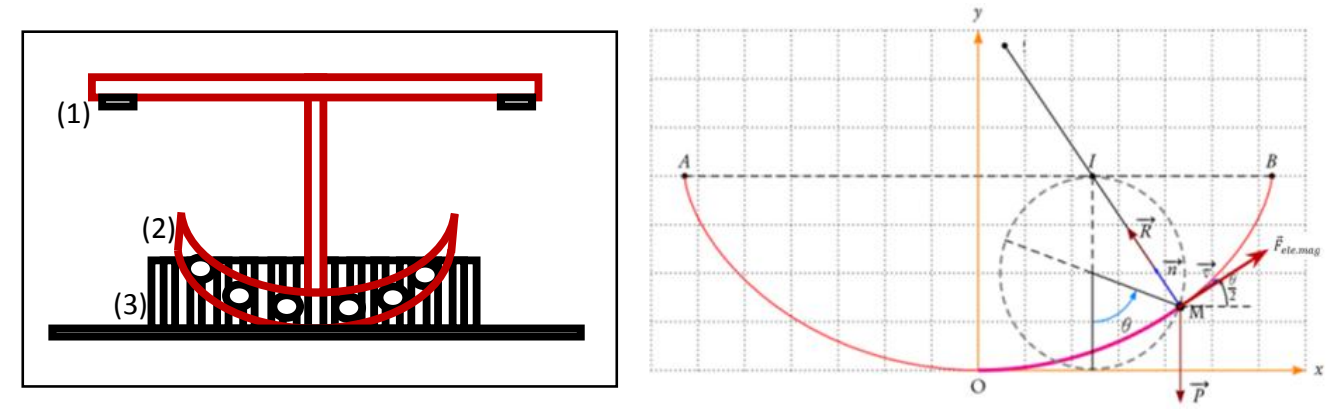

Fig. 2: The Linear Generator that we study

(1) electromagnetic or mechanical system to maintain continuity of motion of the inductor (2)

(2) the inductor of the generator on the coils which are powered by direct current

(3) the armature of the generator, a circuit for closing the magnetic field lines (tooth + winding).

The characteristics of the generator considered are shown in Table (1) summarizes all the electrical and geometrical dimensions.

Table 1. Characteristics of the Generator Considered

\begin{tabular}{|c|c|c|c|c|}
\hline Elements & \multicolumn{2}{|c|}{ Dimensions } & Symbols & Values [mm] \\
\hline \multirow{4}{*}{ Induced } & \multicolumn{2}{|c|}{ Length } & $\mathrm{L}$ & 175 \\
\hline & \multicolumn{2}{|c|}{ Width } & 1 & 30 \\
\hline & \multirow{2}{*}{\multicolumn{2}{|c|}{$\begin{array}{l}\text { Height } \\
\text { Number of Slot }\end{array}$}} & $\mathrm{h}$ & 70 \\
\hline & & & $\mathrm{m}$ & 12 \\
\hline \multirow{5}{*}{$\begin{array}{l}\text { Inductor } \\
\text { (6 Coils) }\end{array}$} & \multirow{3}{*}{ Coil } & Length & $\mathrm{L}_{0}$ & 26 \\
\hline & & Diameter & $\mathrm{D}_{0}$ & 14 \\
\hline & & Diameter of conductor & $\mathrm{d}$ & $\ldots$ \\
\hline & \multirow[t]{2}{*}{ Core } & Length & $\mathrm{L}_{1}$ & 37 \\
\hline & & Diameter & $\mathrm{D}^{\prime}$ & 12 \\
\hline Gap & \multicolumn{2}{|c|}{ Thickness } & $\delta$ & 3.5 \\
\hline
\end{tabular}

\section{The Primary Element Of The Inductor}

A linear generator is a coil with a resistor $R$, the coil may slide without friction in a space between two winding (armature) is moving as a semicircle (Cycloid). the coil is supplied (the coils) to direct current and a constant rate imposed when there is presence of a magnetic field $\vec{B}$. The electromotive force in the two faces of the armature winding will generate an current I armature. And to keep the set of coils at a constant rate (steady state), before we apply an external force perpendicular to the magnetic force induced.

And to get the best results, follow the following steps:

\subsection{SIZING OF THE COIL}

The determination of the design is a great interest for the design of electrical machines. Indeed, the design and evaluation of a coil linear generator based on knowledge of the magnitudes of electromagnetic fields, and among these quantities, magnetic field induction is their practical value is between 1.4 and $1.6 \mathrm{~T}$ to avoid the saturation of the magnetic circuit, and the current density $J$ is also practical value is between 5 and $7 \mathrm{~A} / \mathrm{mm}^{2}$ to avoid overheating of the coil.

So the choice we give is as follows:

$S_{b}($ the wound area $)=(\mathrm{H} * \mathrm{~L})(\mathrm{mm})$

$J_{e x t}=6 \mathrm{~A} / \mathrm{mm}^{2}$ 
From these data it may be added to the conductive member surface, the current that can be injected into this area, and the number of turns of the winding.

And in the following table (2) diameters of winding son and that corresponding currents on site :

Table 2. Diameters of winding and the currents corresponding

\begin{tabular}{|l|l|l|l|}
\hline$\Phi$ wire $\mathrm{D} / \mathrm{mm}$ & For $\mathrm{J}=5 \mathrm{~A} / \mathrm{mm}^{2}$ & For J=6A/mm & For J=7A/mm \\
\hline 0.5 & 0.98 & 1.176 & 1.372 \\
\hline 0.7 & 1.925 & 2.31 & 2.695 \\
\hline 0.9 & 3.18 & 3.816 & 4.452 \\
\hline 1 & 3.925 & 4.71 & 5.495 \\
\hline 1.5 & 8.835 & 10.602 & 12.369 \\
\hline 2 & 15.7 & 18.84 & 21.98 \\
\hline
\end{tabular}

Where : $J_{\text {ext }}=I / S_{e}$

$$
N=S_{b} / S_{e}
$$

$I:$ The amperage we inject

$S_{e}:$ Surface conductive member

$N$ : The number of turns

$S_{b}:$ wound area, AN : $S_{b}=(14 * 26)$

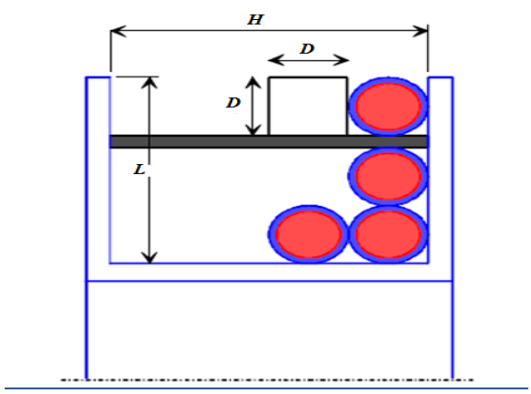

Fig. 3: Cutting a half coil

\section{2 ELECTROMAGNETIC FORMULATION}

For the study of electromagnetic phenomena in electrical machinery Maxwell's equations are used, because they reflect the interactions between electrical and graders take shape as a repository associated with the medium under study.

$$
\operatorname{rot} \vec{H}=\vec{J}+\frac{\partial \vec{D}}{\partial t}, \operatorname{rot} \vec{E}=-\frac{\partial \vec{B}}{\partial t}, \operatorname{div} \vec{B}=0, \operatorname{div} \vec{D}=\rho,
$$

Supplemented by specific relations materials:

$$
\vec{B}=\mu_{0} \mu_{r} \vec{H}, \vec{D}=\varepsilon \vec{E}, \vec{J}=\sigma \vec{E}
$$

For a direct current $\frac{\partial \vec{D}}{\partial t}$ is zero [4], [5], [6].

Using the Model Magneto dynamics is answered in the study of electrical machines. For the numerical computation, there are several formulations such as formulation $\vec{A}$, and in this model we have chosen the use of this formulation with the many benefits:

- this is the most used and it reduces the number of unknowns;

- it can impose electric by the coils;

- knowledge of any physical quantity can be deducted.

We have :

Obtained :

$$
\operatorname{rot} \vec{E}=-\frac{\partial \vec{B}}{\partial t} / \vec{B}=\operatorname{rot} \vec{A}
$$

$$
\operatorname{rot} \vec{E}=-\frac{\partial}{\partial t}(\operatorname{rot} \vec{A})=-\operatorname{rot}\left(\frac{\partial \vec{A}}{\partial t}\right) \Rightarrow \operatorname{rot}\left(\vec{E}+\frac{\partial \vec{A}}{\partial t}\right)=\overrightarrow{0}
$$


$\vec{E}+\frac{\partial \vec{A}}{\partial t}$ is a conservative field, so it is derived from a scalar electric potential $U$, such that :

$$
\vec{E}+\frac{\partial \vec{A}}{\partial t}=-\overrightarrow{\operatorname{grad}} U \Rightarrow \vec{E}=-\left(\frac{\partial \vec{A}}{\partial t}+\overrightarrow{\operatorname{grad}} U\right)
$$

But in this model there is a shift in the $\vec{\vartheta}$ speed of the inductor coil in a magnetic induction field $\vec{B}$ gives rise to induced current density $\vec{J}$ which is, according to the law of Laplace:

$$
\vec{J}=\sigma \vec{\vartheta} \wedge \vec{B}
$$

We must take into account in the previous model, where the induced currents $\sigma \partial \vec{A} / \partial t$ must be replaced by:

$$
\sigma \frac{\partial \vec{A}}{\partial t}+\sigma \vec{\vartheta} \wedge \vec{B}=\sigma \frac{\partial \vec{A}}{\partial t}+\sigma \vec{\vartheta} \wedge \operatorname{rot} \vec{A}
$$

So according to the calculations is added :

$$
\operatorname{rot}\left(\frac{1}{\mu} \operatorname{rot} \vec{A}\right)+\sigma \frac{\partial \vec{A}}{\partial t}+\sigma \overrightarrow{\operatorname{grad}} U-\sigma(\vec{\vartheta} \wedge \operatorname{rot} \vec{A})=\overrightarrow{J_{\text {ext }}}
$$

\section{3 SOLVING FLOWCHART}

The organization is the image of the general organization of a computer program by Finite Elements. The sequential workflow can distinguish three main groups :

- preprocessor : commonly called mesh, responsible for generating the data needed to solve;

- processor resolution : called solver;

- the postprocessor operating results.

These three modules and using techniques of Computer Aided Design (CAD): Write the domain geometry, physical properties and boundary conditions and perform a manual cutting or with assistance from the program, then solve equations of the element assembly, and provides a set of results, and at the end the results stored in an output file that switches form a physical quantity.

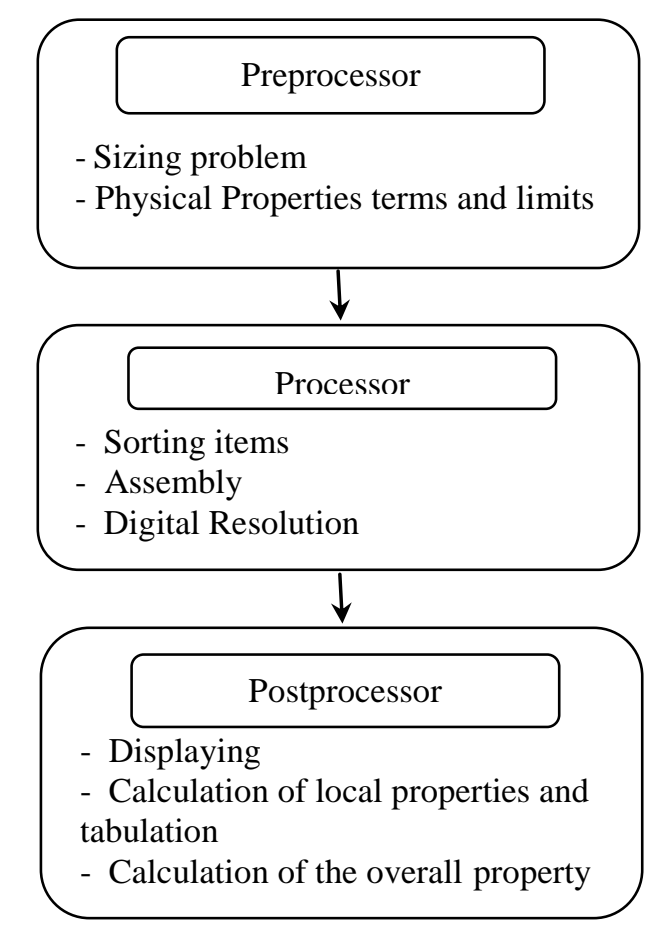

Fig. 4: Organization of software 


\section{4 USEFUL RESOLUTION}

Comsol Multiphysics (FEMLAB) is a software for solving differential model (pde), multiphysics (electromagnetic, mechanical, structural, thermal, ...) by the finite element method with an unlimited number of different physical interaction between many steps. Using this software has many benefits including benefits: contains most of equations using a graphical interface, little direct programming and MATLAB interface possible [7], [8].

after some applications and examples, add the method that allows us to achieve the solution of electromagnetic problems.

So, an analysis was made for three examples :

- At first, it will characterize the magnetic field produced by a single coil which lies between the two windings of the armature of the generator.

- Secondly, we examine the association of six fixed coils.

- Finally, six coils in motion, we exploit this experience to deduce the magnitude of the magnetic field and additive and it creates for the electromotive force that we want the generator.

Figures (5. a), (5. b) And (5. c) Show the magnetic vector potential $\vec{A}$, distribution and induction of the magnetic field $\vec{B}$ for a single coil.

These figures show the magnetic vector potential and these contours and the magnetic induction in the coil, the air gap and of the winding of the armature. The value of the induction is reasonable because we know that the linear machine function in saturation mode, but we tried to avoid it and the heating of the machine to determine good values of current density and the sizing of coils.

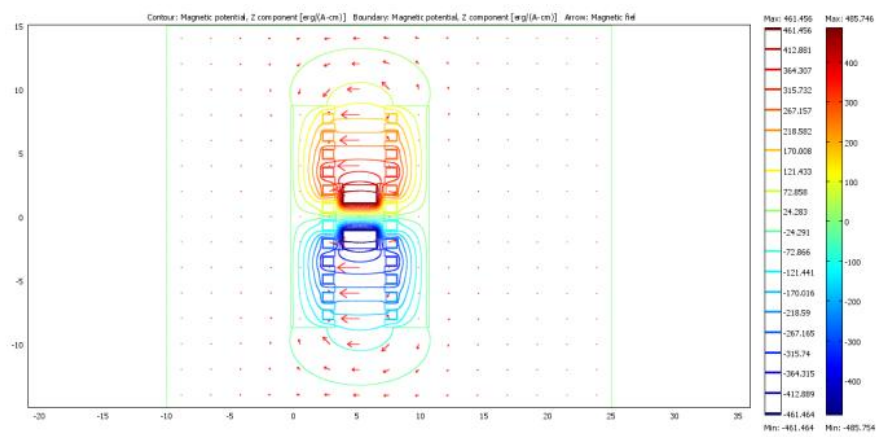

Fig. 5. a: The distribution of the magnetic vector potential by a single coil
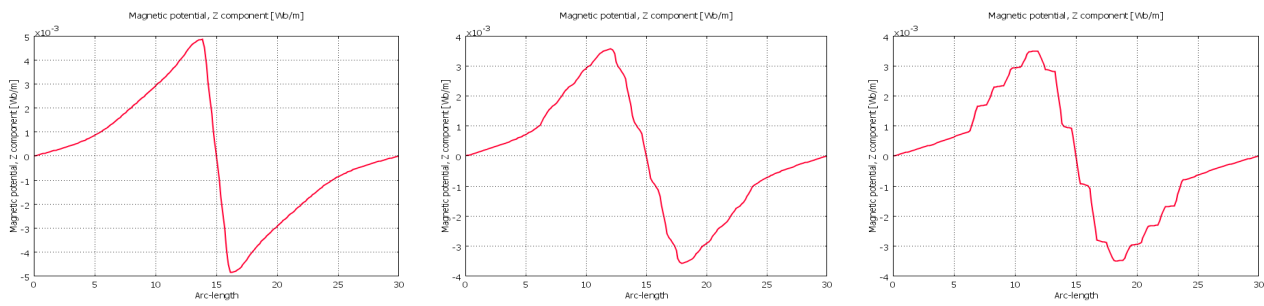

Fig. 5. b: The values of the magnetic vector potential in the coil, the air gap and the armature winding
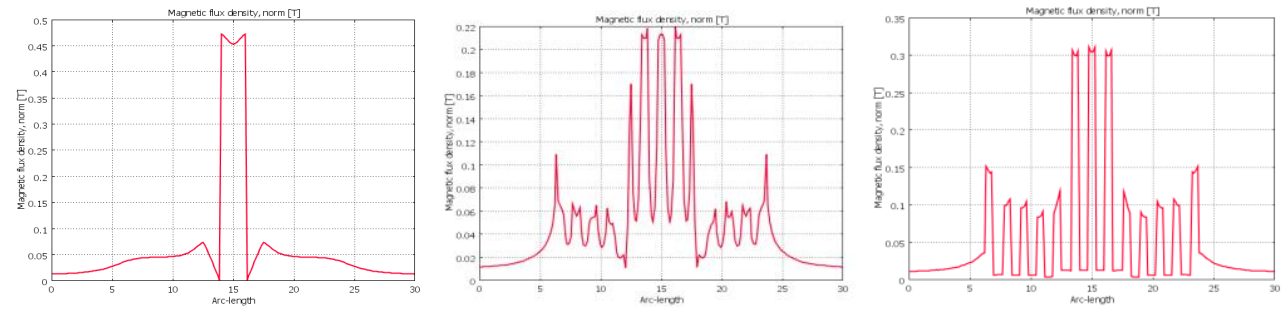

Fig. 5. c: The values of magnetic induction in the coil, the air gap and the armature winding 


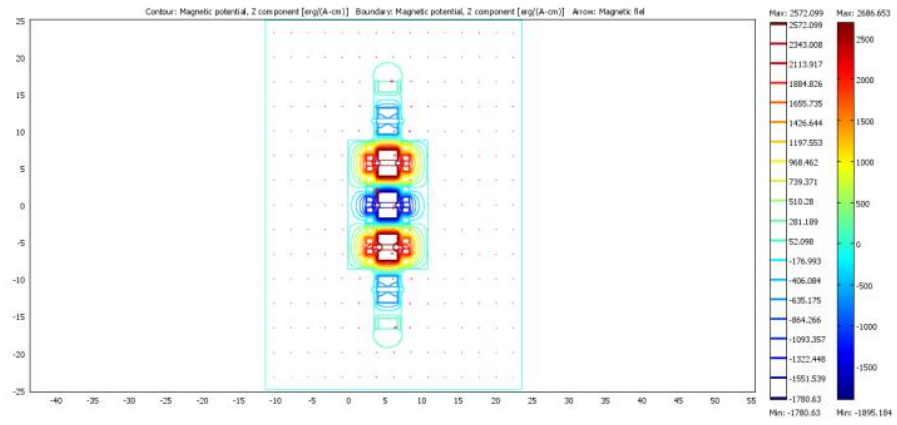

Fig. 6. a: The distribution of the magnetic vector potential by six fixed coils
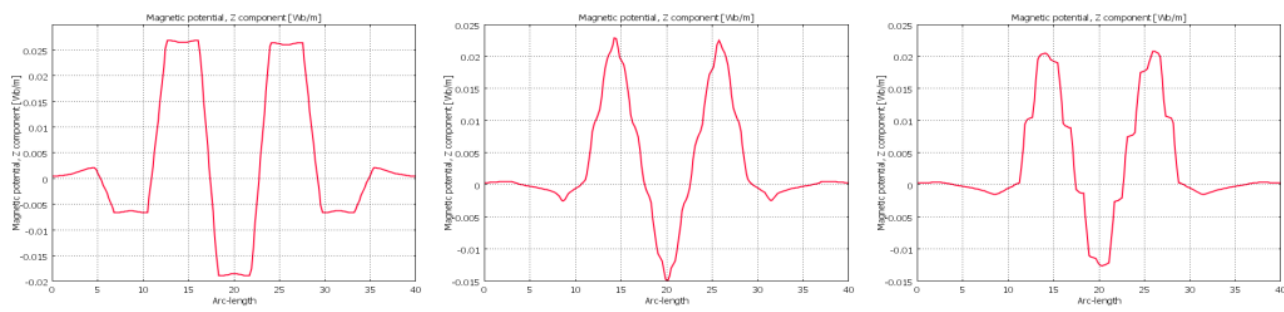

Fig. 6. b: The values of the magnetic vector potential in the coil, the air gap and the armature winding
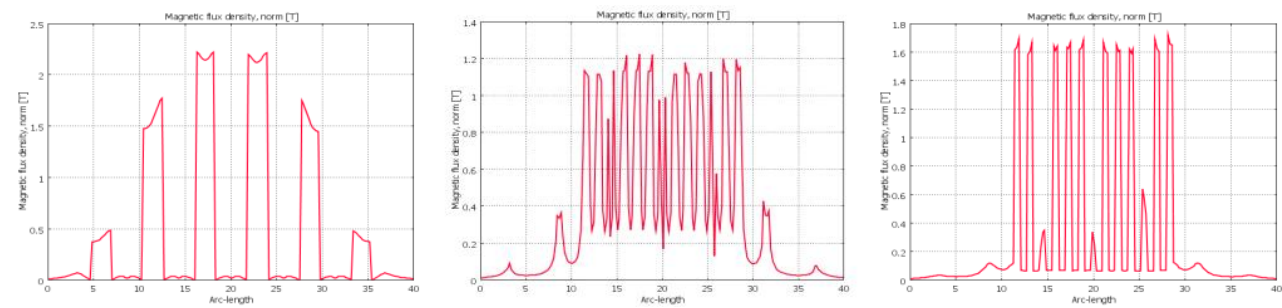

Fig. 6. c: The values of magnetic induction in the coil, the air gap and the armature winding

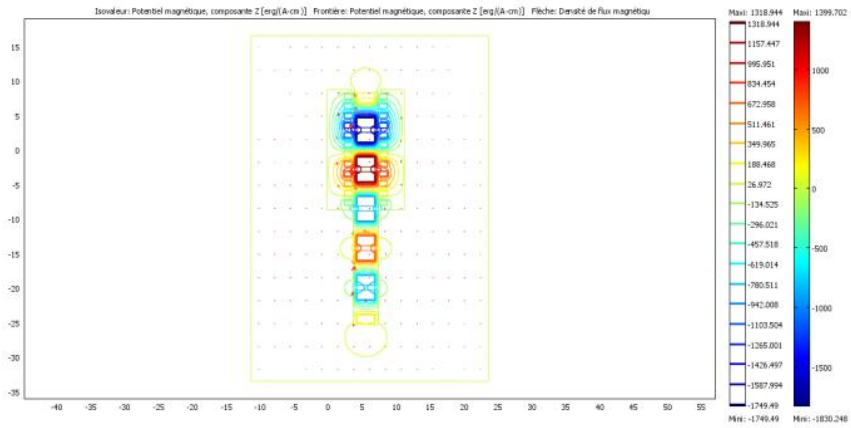

Fig. 7. a: The distribution of the magnetic vector potential by six coils in motion
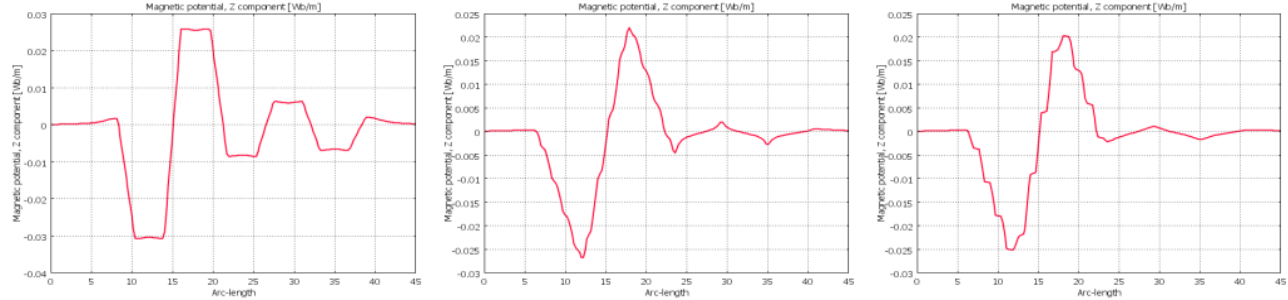

Fig. 7. b: The values of the magnetic vector potential in the coil, the air gap and the armature winding 

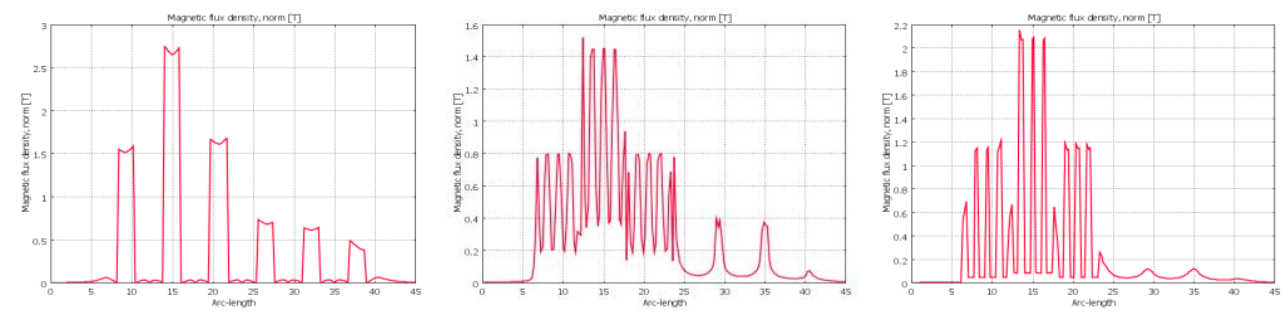

Fig. 7. c: The values of magnetic induction in the coil, the air gap and the armature winding

Therefore, the figures (5), (6) and (7) show the influence of:

- Number of coils used in the inductor to obtain the value of the electromotive force that we want.

- Measuring the location of the field and the magnetic induction.

\section{Conclusion}

This article focuses on how to analyze and solve the problem of the magnetic field in the inductor of the linear generator using the finite element method, the strategy for Computer Aided Design, and to do this we adds to the electromotive force that need.

- We analyze the relationship between the maximum intensity of the magnetic field and geometric dimensions.

- A comparison was made between dependency of values of the magnetic field of the coils, and locations of measurement.

\section{Acknowledgements}

I thank Dr. D. Hedjazi who suggested the subject of his constant support and the advice and guidance, and thanks also go to Prof. R. Abdessemed, and all those who gave me their help in any way.

Lahouel Dalila was born on April 26, 1981 in Batna, Algeria. She studied the Electrical Engineering at the Faculty of Electrical Engineering from the Batna University, and graduated in 2006. At the moment she is a PhD student of the course of control of Electrical Machines. Faculty of Electrical Engineering, in Batna University; Algeria. d_lahouel@yahoo.fr.

\section{Periodicals:}

\section{REFERENCES}

[1] H. Allag, M. E. H. Latreche, M. R. Mekideche and N. Ikhlef. Axial Magnetic Forces Comparison in Linear and Nonlinear Model of Ferromagnetic Core of Magnetic Actuators, Medwell Journals, 2007 (P :1199-1202)

[2] R. Blanchet, F. Gaume et A. Bondeau. Distribution Optimale du Courant dans une Bobine sans Fer pour Mesurer de Susceptibilité Magnétique, Revue de Physique Appliquée Mars, 1973 (83)

\section{Technical Reports:}

[3] Dulpichet Rerkpreedapong. Analyse de Champ et Conception d'un Alternateur Linéaire de Fer Mobile pour l'Usage avec le Moteur Linéaire. Université de Virginie Occidentale. 1999

[4] Bennecib Nedjoua. Contribution a l'Etude d'une Machine MHD a Conduction en vue de son Exploitation sur un Réseau Electrique. Université de Batna. Algérie. 2010

[5] Hedjazi Djemai. Etude et Conception d'un Moteur Linéaire Double Induction. Université de Batna. Algérie. 1994

\section{Articles from Conference Proceedings (published):}

[6] Jawad Faiz, Mahdi Ebrahimi-Salari, and Gh. Shahgholian. Reduction of Cogging Force in Linear Permanent-Magnet Generators. University of Tehran, Tehran, Iran. IEEE Transactions on Magnetics, January 2010

[7] André Buchau, Wolfgang M. Rucker. Analysis of a Three-phase Transformer Using COMSOL Multiphysics and a Virtual Reality Environment. Universität Stuttgart, Stuttgart, Germany

[8] Dudnikov Evgeny, Shmelev Vjacheslav, Walde Bertil. Educational electronic course "Theory of the Electromagnetic Field" on the Basis of the Program Complex COMSOL Multiphysics. Institute of Control Problem, Moscow, Russia, Vladimir State Technical University, Vladimir, Russia 